\title{
Two-Color Green Fluorescent Protein Time-Lapse Imaging
}

BioTechniques 25:838-846 (November 1998)

\author{
Jan Ellenberg, Jennifer Lip- \\ pincott-Schwartz and John \\ F. Presley \\ National Institute for Child \\ Health and Human Develop- \\ ment, NIH, Bethesda, MD, USA
}

\begin{abstract}
The Aequorea victoria green fluorescent protein (GFP) is widely recognized as a powerful tool in cell biology, serving as a vital reporter for monitoring localization and dynamics of intracellular proteins and organelles over time. GFP variants with shifted spectral characteristics have been described and offer enormous potential for double-labeling experiments and proteinprotein interaction studies. However, most GFP variant combinations are not suitable for double-label, time-lapse imaging experiments because of either extremely rapid photobleaching of blue-shifted GFP variants or crossover of their excitation and emission spectra, which must then be computer corrected. Here, we describe the successful use of two photostable spectral GFP variants, W7 and 10C, in dual-color, timelapse imaging of fusion proteins in living cells using either wide-field or confocal microscopy. W7 and 10C were highly photostable during repetitive long-term imaging and were cleanly separated by their different excitation spectra alone with negligible crossover of fluorescence. We present timelapse image sequences of COS-7 cells co-expressing both a marker of the Golgi complex (galactosyl transferase) fused to W7 and a marker of the nuclear envelope (lamin-B receptor) fused to 10C. To our knowledge, these image sequences provide the first simultaneous visualization of Golgi and nuclear envelope membranes in living cells.
\end{abstract}

\section{INTRODUCTION}

New insights into the dynamics and movement of various proteins in living cells have been achieved using green fluorescent protein (GFP) fusions $(1,2,6,10,12,17,18,20,22)$. Such studies typically require time-lapse imaging over periods ranging from minutes to several hours and can involve the collection of more than 100 images from the same cell. The most commonly used fluorescein-like GFPs (with spectral properties similar to the S65T mutant; Reference 7) have proven sufficiently bright and photostable for these purposes. Double labeling cells with GFP variants that fluoresce at different wavelengths $(8,9)$ has only begun to be used for comparing the distribution and dynamics of two different populations of proteins within cells. For this approach, cells are doubly transfected with proteins attached to different GFP variants that have distinct excitation or emission spectra and are imaged with different filter sets. Several groups have successfully distinguished blue-emitting and red-shifted GFP variants in doubly transfected cells (19), but these combinations of spectral GFP variants have proven unsatisfactory for double labeling in time-lapse experiments. This is primarily because the blue-shifted variants that were used are rapidly photobleached, making the acquisition of an extended image series from the same cell impossible.

A previous publication has demonstrated the potential of dual-color, timelapse imaging with GFP (23). In this study, however, there was substantial crossover between the two spectral GFP variants used (W2 and S65T; Reference 9) that had to be corrected after image acquisition. This poses serious problems if the two GFPs are imaged in the same structures and is impractical for large data sets. Moreover, the dualcolor, time-lapse imaging setup used required a system with two separate detectors (such as a confocal microscope), limiting applicability to laboratories with sophisticated imaging systems.

Here, we describe the use of $10 \mathrm{C}$ (which has red-shifted excitation and emission compared with S65T; References 7 and 15) and W7 variants of GFP that are suitable for use in timelapse imaging experiments on conventional wide-field or confocal microscopes without problems of crossover or photobleaching. This approach uses inexpensive GFP/filter combinations for imaging on wide-field microscopes that avoid registration error caused by changing dichroics. Readily available laser/filter combinations for imaging on a confocal microscope are also used. The practicality of these methods is demonstrated with our dual-color, time-lapse analysis of Golgi and nuclear membranes in living cells.

\section{MATERIALS AND METHODS}

\section{GFP Variants}

Descriptions of the five key GFP variants we used and their excitation/ emission (ex/em) wavelengths (in nm) for imaging follow: (i) EGFP (F64L, S65T, H231L; ex/em 488/507), (ii) EBFP (F64L, Y66H, S65T, T145L; ex/em 380/440) both from CLONTECH Laboratories, (Palo Alto, CA, USA), (iii) BFP (F64L, Y66H, Q80R, V163A; ex/em 387/450; Quantum Biotechnologies, Montreal, Canada), (iv) 
10C (S65G, V68L, S72A, T203Y; ex/ em 513/527; Reference 15) and (v) W7 (Y66W, Q80R, N146I, M153T, V163A, N212K; ex/em 433/475). Variant 10C was made by site-directed mutagenesis using the EGFP sequence as a template and introduced back into the pEGFP$\mathrm{N} 1$ vector replacing the original EGFP. Variant W7 was obtained from R. Tsien (University of California at San Diego) and contains the additional Q80R mutation that does not significantly change the published spectral properties (compare Figure 2 and Reference 9).

\section{Spectral Analysis}

Variant 10C was subcloned into pET-28a (Novagen, Madison, WI, USA) using a polymerase chain reaction (PCR)-generated NdeI site at the ATG and the NotI site of the pEGFPN1 backbone. W7 cDNA was expressed from pRSET B (a gift from R. Tsien). GFP variants were expressed in pET Host Strain BL21 (DE3) (Novagen), and pre-cleared lysates were diluted in $50 \mathrm{mM}$ Tris- $\mathrm{HCl}, \mathrm{pH} 8.0$ before measuring spectra on a DeltaScan Spectrofluorometer (Photon Technology International, Carrboro, NC, USA). For the W7 excitation spectrum, emission was held constant at $505 \mathrm{~nm}$, and for the emission spectrum, excitation was held constant at $415 \mathrm{~nm}$. The excitation spectrum of variant $10 \mathrm{C}$ was measured with constant emission at 550 $\mathrm{nm}$, and the emission spectrum was obtained with constant excitation at 488 $\mathrm{nm}$. Spectra were collected in 1-nm steps with averaging over $2 \mathrm{~s}$. Control measurements were performed with values closer to the excitation and emission maxima and resulted in identical spectra. Spectra were corrected for the autofluorescence of $E$. coli lysates measured in non-expressing cultures at the same cell density. After correction, spectra were normalized to their respective peaks as $100 \%$ for comparison.

\section{Targeted Expression in Mammalian Cells}

GT-W7 contains the first 60 amino acids (aa) (analogous to GT-GFP in Reference 2) of human galactosyl transferase placed upstream of W7 in a pEGFP-N1-based vector (CLONTECH
Laboratories) using $\mathrm{SacI}$ and SacII restriction sites. This generated a 13-aa spacer (STVPRARDPPVAH) between GT and W7. The human lamin B receptor (LBR)-10C fusion protein contains the first 238 aa of human lamin B receptor (analogous to Reference 6) placed upstream of GFP variant 10C in a pEGFP-N1-based vector, and was cloned using the BamHI site to create a 4-aa spacer (PVAH).

\section{Filter Sets and Microscope Systems}

Most experiments used a custombuilt, wide-field fluorescence microscope with a cooled charge-coupled device (CCD) camera (Photometrics, Tucson, AZ, USA) and a KAF 1400 pixel Kodak chip (Eastman Kodak, Rochester, NY, USA). The microscope setup was as described by Sciaky et al. (21). Briefly, two computer-controlled filter wheels immediately followed a $100-\mathrm{W}$ mercury lamp and computercontrolled shutter in the light path, the first wheel contained a graded series of neutral-density filters, while the second contained excitation filters. Lamp, filter wheel and shutters were all from Ludl Electronic Products (Hawthorne, NY, USA). The following excitation filters were used: (i) W7 bandpass (BP) $414 \pm$ $15 \mathrm{~nm}$, (ii) 10C BP $487 \pm 15 \mathrm{~nm}$, (iii) EBFP BP $390 \pm 20 \mathrm{~nm}$ and (iv) BFP BP $390 \pm 20 \mathrm{~nm}$. The common dichroic was SP 505 (all from Chroma Technology, Brattleboro, VT, USA), whereas the common emission filter (placed immediately after the dichroic in the emission light path) was BP $535 \pm 27.5$ (Omega Optical, Brattleboro, VT, USA). Shutters and filter wheels were controlled by a LEP box (Ludl Electronic Products) and Macintosh ${ }^{\circledR}$ Quadra running IPLab Spectrum ${ }^{\mathrm{TM}}$ Software (Scanalytics, Vienna, VA, USA).

The confocal laser scanning microscope system was based on a Zeiss LSM 410 (Carl Zeiss, Thornwood, NY, USA) equipped with a triple-line Series 43 488/568/647-nm Kr/Ar laser (Omnichrome, Chino, CA, USA) and an Enterprise II $\mathrm{Kr}$ laser optimized for the 413-nm Kr line (Coherent, Santa Clara, CA, USA). To image W7, we used the 413-nm $\mathrm{Kr}$ laser line in conjunction with a FT-440 dichroic and a LP-460 emission filter (both from Chroma 
Technology). To image variant $10 \mathrm{C}$, the 488-nm laser line with a standard dualFT488/568 dichroic and a BP 515-560 (both from Carl Zeiss) was used.

\section{Photobleaching}

To compare photobleaching properties of the GFP variants, they were expressed transiently in COS-7 cells under the control of a cytomegalovirus (CMV) promoter ( $\mathrm{pEGFP}-\mathrm{N} 1-$ based vectors for EGFP, EBFP, 10C and W7; pQBI50 for BFP) as soluble proteins. Cells were imaged live in phosphate-buffered saline (PBS), pH 7.4 at $37^{\circ} \mathrm{C}$ using a Model ASI 400 Air Stream Stage Incubator (Nevtek, Burnsville, VA, USA) with continuous full illumination from a 100W HBO mercury bulb on the cooled CCD wide-field microscope using a $100 \times 1.4$ NA Planapochromat oil immersion objective (Carl Zeiss) for most efficient photobleaching. Twelve-bit digital whole-cell images were acquired with a series of 1-s exposure times at 6.7-s intervals for a total imaging time of 402 s. Fluorescence intensity was quantified in representative regions of interest (ROI), background subtracted and plotted over time using IPLab Spectrum software. The initial intensity was normalized to 100 for comparison. For each GFP variant, three independent cells were averaged to determine photobleaching rate constants. For EBFP, only datapoints above 30 (after normalization) were considered for rate constant fitting, below that data were too close to background. Relative excitation energy at three different wavelengths (390, 414 and $487 \mathrm{~nm}$ ) was measured using a photomultiplier tube (Carl Zeiss) attached above the specimen plane, and photobleaching rates were corrected for the differences in intensities. All GFP variants were measured on the same day in a continuous experiment to ensure constant energy levels of the excitation source. The photoconversion that has been reported for wild-type (WT) GFP (16) could not be detected for either 10C or W7 variants whose photobleaching curves were fit accurately by a single exponential decay.

\section{Time-Lapse Imaging}

GT-W7 and LBR-10C were co-expressed transiently in COS-7 cells, and cells were imaged in chambered cover glasses (LabTek, Naperville, IL, USA) containing RPMI without phenol red, and supplemented with $10 \%$ fetal calf serum (FCS), $25 \mathrm{mM}$ HEPES-KOH, $\mathrm{pH}$ 7.4, $2 \mathrm{mM}$ glutamine, $100 \mu \mathrm{g} / \mathrm{mL}$ penicillin and $100 \mathrm{U} / \mathrm{mL}$ streptomycin (all from BioFluids, Rockville, MD,

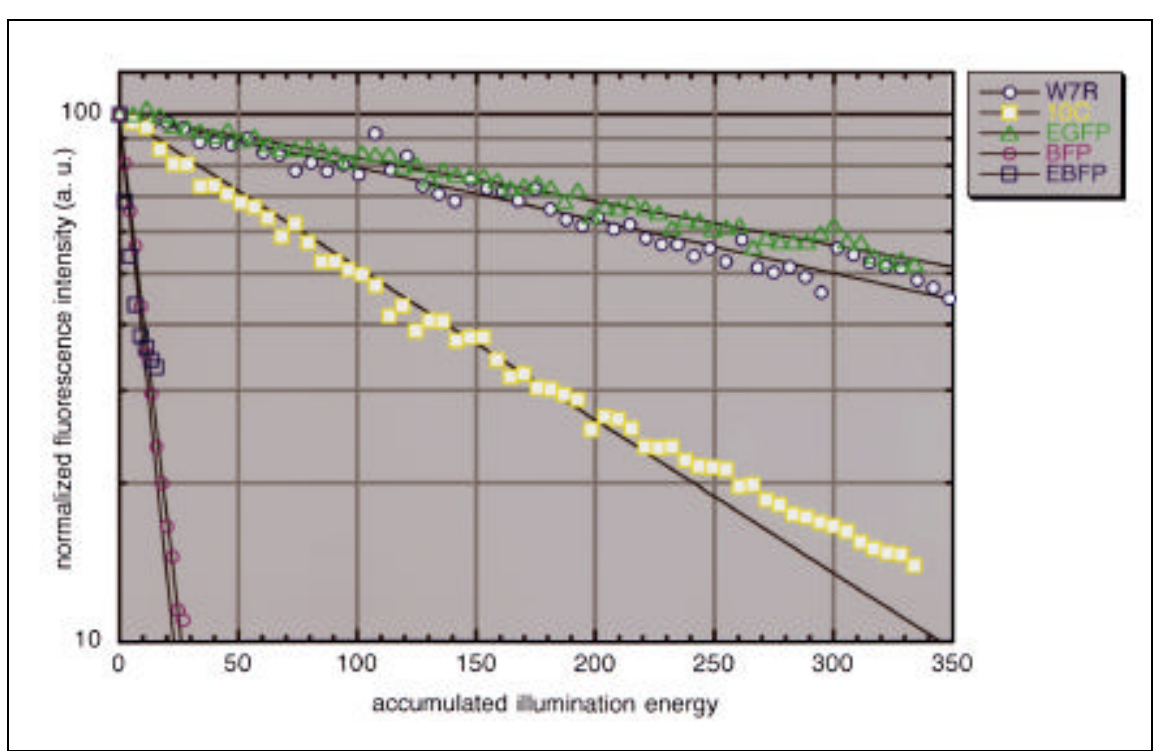

Figure 1. Photobleaching properties of GFP variants. Fluorescence intensity (log scale) as a function of accumulated illumination energy (linear scale) of soluble GFP variants expressed in COS-7 cells; EGFP (green triangles), W7 (blue circles), 10C (yellow squares), BFP (purple circles) and EBFP (blue squares). Initial intensities were normalized to 100 , and data were fit to a single exponential decay function (solid lines) to determine rate constants (see text).
USA) at $37^{\circ} \mathrm{C}$.

For imaging of brefeldin A (BFA)treated cells, images of GT-W7 (excitation 399-429 nm; exposure, $1.5 \mathrm{~s}$ ) and LBR-10C (excitation 472-502 nm; exposure, $0.5 \mathrm{~s}$ ) were captured sequentially, at 5-s intervals, on a wide-field microscope-CCD camera setup. These image pairs were taken every $30 \mathrm{~s}$, starting two min after the addition of 10 $\mu \mathrm{g} / \mathrm{mL}$ BFA (Epicentre Technologies, Madison, WI, USA) with attenuated illumination (39.8\% transmission), using a $100 \times 1.4$ NA Planapochromat oil immersion objective (Carl Zeiss) on the microscope system described above. Images were converted to 8 bit, background was subtracted and display was either side by side using IPLab Spectrum or in color overlay using Adobe ${ }^{\circledR}$ Photoshop $^{\circledR} \quad$ (Adobe Systems, San Jose, CA, USA).

Untreated cells were imaged on the confocal laser scanning microscope (CLSM) system described above using $30 \%$ or $15 \%$ laser power and $3 \%$ or $1 \%$ transmission (413/488 nm), respectively. A series of $6 \mathrm{z}$-sections $2.5 \mu \mathrm{m}$ apart was collected every $10 \mathrm{~min}$ for both W7 and 10C after autofocussing on the cover-glass surface in reflection mode using a $100 \times$ Planapochromat 1.4 NA oil immersion objective over a total time of 9 h 30 min (336 image pairs). Eight-bit images were background-subtracted and z-stacks projected at $0^{\circ}$ and $90^{\circ}$ using NIH Image 1.62 Software (http://rsb.info.nih.gov/nih-image). The resulting projected images were then color-merged for display using Adobe Photoshop.

Rescue of the photobleached "dark state", by far blue irradiation that has been described for single molecules of other T203F/Y GFP variants (3), could not be detected for 10C using either the wide-field or confocal microscope setup in live cells. In contrast to those mutants, $10 \mathrm{C}$ is lacking a minor excitation peak (Figure 2).

\section{Crossover Measurements}

GT-W7 and LBR-10C were expressed separately in COS-7 cells and imaged in PBS as described above for both microscope systems collecting sequential images at both excitation wavelengths. ROIs in brightly labeled 
structures were quantified, background-subtracted and then compared with the corresponding ROIs in the paired image. For each GFP, seven independent ROIs were used for quantification. For measurements in E. coli, W7 and 10C were expressed separately as native proteins, and crossover was determined in the same manner.

\section{RESULTS AND DISCUSSION}

In our selection of GFP variants for double labeling, we sought a pair where each variant was highly photostable and could be discriminated on the basis of its different excitation spectra alone. This pair would then be suitable for wide-field microscopes that have computer control of excitation sources, but no control of dichroics and emission filters. We chose W7 (9) and 10C (15) to investigate as a double-label pair because W7 was found to be the most photostable of all available blue-shifted variants and had a short-wavelength excitation $(433 \mathrm{~nm})$. Variant $10 \mathrm{C}$ by contrast had, to our knowledge, the longest-wavelength excitation and emission peaks of any published GFP variant yet $(513 / 527 \mathrm{~nm})$. It was also photostable.

To document the photostability of the W7/10C pair quantitatively, we measured their rate of photobleaching and compared it with the photobleaching rates of three commonly used green- and blue-GFP variants available commercially: EGFP, EBFP (CLONTECH Laboratories) and BFP (Quantum Biotechnologies). Images were collected from COS-7 cells separately expressing each of the GFP variants as a soluble, cytosolic protein. Cells were illuminated continuously with the appropriate excitation for the various fluorophores, and their brightness was analyzed as a function of accumulated illumination energy (Figure 1). The loss of fluorescence intensity for each of the five GFP variants that were ana- lyzed could be fit by a single exponential decay. The rate constants were normalized to EGFP (the most photostable variant tested) as $1.0 \pm 0.3$. W7 photobleached with a rate constant of $1.3 \pm$ 0.2 and was almost as photostable as EGFP. Variant 10C photobleaching was only $3 \times$ this rate $(3.5 \pm 0.6)$, while both of the "Blue Fluorescent Proteins" were photobleached at $40-60 \times$ this rate (BFP $46.3 \pm 6.9$ and EBFP $57.0 \pm$ 10.4). Photobleaching was normalized to total energy transmitted (which varied between the different filters) but not to total energy absorbed (which depends on the extinction coefficient of the GFP molecule at its excitation wavelengths). Therefore, EBFP and $\mathrm{BFP}$ (which have very low extinction coefficients compared with other GFP molecules) are even more susceptible to bleaching than shown here. Thus, although previously described "Blue Fluorescent Proteins" $(8,13)$ and commercially engineered variants (e.g., EBFP and BFP) have shown good spectral
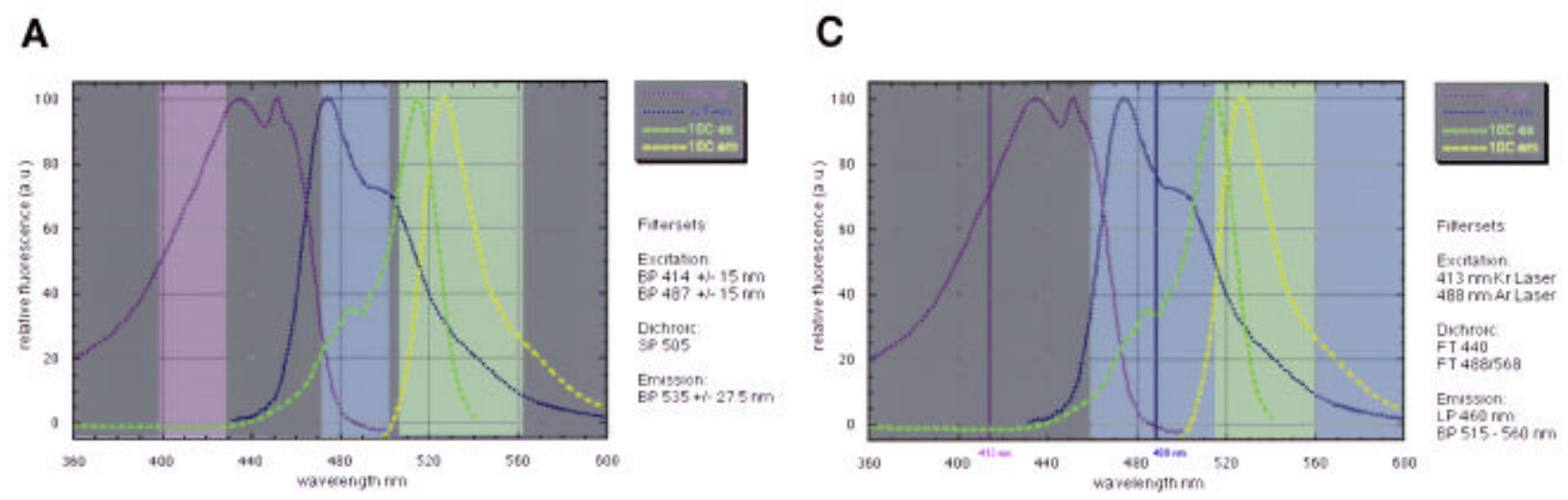

\section{B}
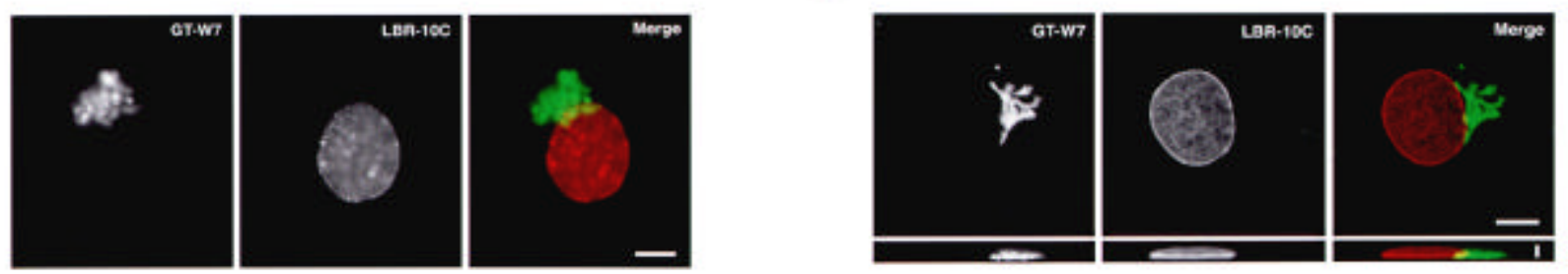

Figure 2. Comparison of W7- and 10C-fluorescence spectra. (A,B) Cooled CCD wide-field microscope setup. (A) Fluorescence spectra of W7 (excitation, purple dotted line; emission, blue dotted line) and 10C (excitation, green dashed line; emission, yellow dashed line). Spectra were normalized to peak fluorescence as 100 arbitrary units (a.u.). Filter bandwidths for W7 excitation (purple shading), 10C excitation (blue shading) and common emission (green shading) are indicated. (B) Double-label images of live COS-7 cells co-expressing GT-W7 (left panel, Golgi complex), LBR-10C (middle panel, nuclear envelope) and a pseudocolor-merged image (right panel). Bar represents scale of $10 \mu \mathrm{m}$. (C,D) CLSM setup. (C) see Panel A for line plots. Laser lines for W7 (413 nm, purple line) and 10C (488 nm, blue line) and emission filters for W7 (blue shading) and 10C (green shading overlaid on top of blue) are indicated. (D) Double-label images of the same cells as in Panel B. Images are projections of z-stacks at $0^{\circ}$ and $90^{\circ}$, respectively. Bars in $\mathrm{x}$ and $\mathrm{z}$ represent $10 \mu \mathrm{m}$ per scale division. 
separation from green-emitting GFP variants, they are unlikely to be useful in time-lapse imaging experiments that use high light intensities delivered by high-numerical-aperture objectives, because they are dim and photobleach extremely rapidly.

Having established that the W7/10C pair was highly photostable, we next investigated whether their spectra could be cleanly separated for dual-label imaging on either a wide-field fluorescence microscope with a cooled CCD camera or a CLSM (see Materials and Methods). As discussed below, each of these microscope systems was able to easily discriminate W7 from 10C fluorescence with minimal crossover of fluorescence when imaged with appropriate filter combinations and/or laser lines.

Side-by-side comparison of the excitation and emission spectra of W7 and 10C (Figure 2A), indicated that a filter set consisting of a common dichroic and emission filter with only different excitation filters would allow discrimination between these variants on a wide-field fluorescence microscope (see Materials and Methods and Figure 2A). Although a significant portion of the emission spectrum of W7 was not used when imaging with the common dichroic, the W7 signal was still easily detected. Emission acquisition from 10C was nearly optimal. A dual dichroic was not feasible given the excitation and emission spectra of the two proteins (see Figure 2). Variations on this filter set are possible. Given the bright signal we obtained from $10 \mathrm{C}$, it would likely excite well with a narrower filter $(2.5-5.0 \mathrm{~nm})$ centered on 485 $\mathrm{nm}$. This, combined with a 495-nm dichroic would allow capturing more of the W7 emission (trading off against poorer excitation of the 10C). Alternatively, two separate filter blocks (with separate excitation filters, dichroics and emission filters for $\mathrm{W} 7$ and 10C) can be used if the microscope design allows collecting image pairs without registration shift (as on a confocal microscope) or if the investigator is willing to correct registration errors manually.

To determine if 10C and W7 could be used as reporter molecules to distinguish different proteins in living mammalian cells, we fused $10 \mathrm{C}$ to the inner nuclear membrane marker lamin B re- ceptor (LBR-10C) and W7 to the Golgi complex marker galactosyl transferase (GT-W7). After co-transfection of both chimeras into COS-7 cells, images were collected on the wide-field, fluorescence microscope using the $10 \mathrm{C}$ and W7 excitation filters and the common dichroic and emission filter. Figure $2 \mathrm{~B}$ shows that the nuclear membrane (red, LBR-10C) and the Golgi complex (green, GT-W7) were clearly and separately visualized in the image pair. In these cells, the Golgi complex appeared as a tight cluster of membranes positioned to one side of the nucleus. As previously shown, LBR-10C was nonuniformly distributed across the nuclear envelope surface (6). Negligible crossover of $10 \mathrm{C}$ and $\mathrm{W} 7$ fluorescence was observed between the two imaging channels (Figure 2B). Variant 10C showed $3.79 \% \pm 0.12 \%$ signal if excited with the W7 BP, and W7 showed $2.95 \pm 0.27 \%$ if excited with the $10 \mathrm{C}$

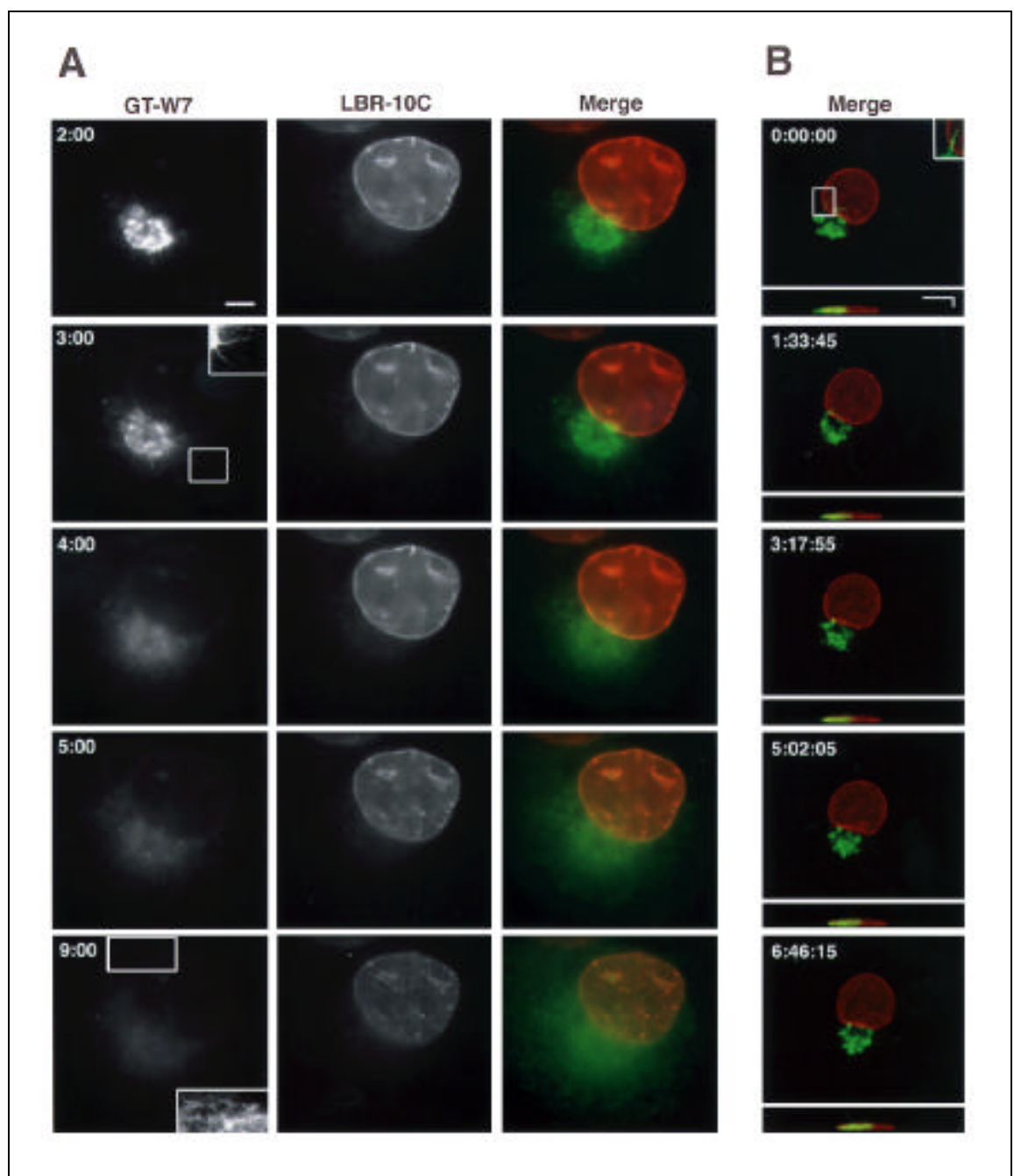

Figure 3. Time-lapse imaging of the Golgi complex and nuclear envelope in live cells. (A) Doublelabel, time-lapse sequence from COS-7 cells co-expressing GT-W7 and LBR-10C treated with BFA was acquired on a cooled, CCD wide-field fluorescence microscope. The first frame shown was taken $2 \mathrm{~min}$ after adding BFA, when the Golgi membranes first started to tubulate. Representative frames are shown over the course of 7 min until the Golgi was redistributed completely into the ER, while the nuclear envelope was unaffected. Insets: the first inset shows a contrast-enhanced magnification of Golgi membrane tubules, the second a part of the ER reticulum. Insets are contrast-enhanced to show dimly labeled structures. Bar represents a 10- $\mu \mathrm{m}$ scale division. (B) Time-lapse sequence from the same cells as in Panel A without drug treatment acquired on a CLSM. Z-stacks of 6 sections were acquired at $10 \min 25 \mathrm{sin}$ tervals for a total of $9 \mathrm{~h}$ and $30 \mathrm{~min}$ (336 image pairs). Representative images shown are projections of $\mathrm{z}$ stacks at $0^{\circ}$ and $90^{\circ}$, respectively. Bars in $\mathrm{x}$ and $\mathrm{z}$ represent a $10-\mu \mathrm{m}$ scale division. Inset is contrast enhanced to emphasize dimly labeled Golgi tubules. 
BP. The latter was most likely due to lysosomal autofluorescence in the Golgi area. Such autofluorescence can be detected even in non-transfected cells excited with the W7 filter. Negligible crossovers of the GFP variants were also found for the bacterially expressed native proteins (data not shown).

Given the excellent discrimination of the W7- and 10C-GFP pair using wide-field fluorescence microscopy, we examined whether they could also be used for double-label imaging on a CLSM. The CLSM is a powerful tool for live-cell imaging, because it is able to capture small subcellular details and dynamics due both to its superior resolution in $z$-and rapid image acquisition. Many commercial CLSMs allow computer control of dichroics and emission filters in addition to control of different laser lines. Figure $2 \mathrm{C}$ illustrates how we optimized these components to discriminate W7 from 10C fluorescence. Whereas $10 \mathrm{C}$ is readily excited by the standard 488-nm Ar laser line and was detected with a FT 488/568 dichroic and a 515-560 BP emission filter, W7 requires excitation in the short wavelength visible range that is not available with conventional $\mathrm{Ar}$ or $\mathrm{Kr} / \mathrm{Ar}$ lasers. We therefore used an Enterprise II 413$\mathrm{nm} \mathrm{Kr}$ laser to excite W7 and a FT 440 dichroic in conjunction with a 460-nm, long-pass emission filter to detect its emission (Figure 2C).

To test this combination of laser lines, dichroics and emission filters on the CLSM, we again imaged COS-7 cells co-expressing LBR-10C and GTW7 (Figure 2D). There was no detectable crossover of $10 \mathrm{C}$ when imaged with the W7 laser/filter configuration, while the signal from $\mathrm{W} 7$ imaged with the $10 \mathrm{C}$ setup was below $1 \%$. The latter was again most likely because of autofluorescence from lysosomes. Brightness of the W7 was improved significantly over the wide-field microscope system because of the optimal emission detection for this variant (compare Figure 2, $\mathrm{A}$ and $\mathrm{C}$ ).

The suitability of the 10C/W7 double-labeling pair for prolonged timelapse imaging experiments using the microscope systems described above was investigated in living COS-7 cells co-expressing GT-W7 and LBR-10C. On the wide-field microscope, cells were imaged during treatment with the drug brefeldin A (BFA), which causes the Golgi complex to undergo profound morphological changes. Within minutes of BFA treatment, Golgi membranes tubulate and fuse with the ER, resulting in a mixing of Golgi proteins with proteins of the ER and nuclear envelope $(11,21)$. Figure $3 \mathrm{~A}$ shows exemplary frames from such a time-lapse experiment over the course of $15 \mathrm{~min}$ (30 image pairs) covering the entire process whereby Golgi membranes tubulate, fuse with and then redistribute into the ER. Untreated cells co-expressing GTW7 and LBR-10C could be imaged over the course of 30 min (120 image pairs) without dramatic loss in signal or damage from the illumination through the W7 filter (data not shown). The brightness of LBR-10C was comparable with the signal obtained from an identical fusion to EGFP. The brightness of GT-W7 was significantly lower than from the corresponding EGFP construct (data not shown) due to suboptimal emission detection for W7 by the common dichroic/emission filter (Figure 2A). Nevertheless signal intensity was bright enough to detect small structures such as Golgi membrane tubules and the dimly labeled ER after redistribution by BFA (Figure 3A, insets). LBR-10C brightly outlined the nuclear envelope over the course of the experiment, suggesting that despite some mixing of its surface with Golgi membrane proteins, its overall morphology was unaffected by the BFA treatment.

On the CLSM system, untreated COS-7 cells expressing LBR-10C/GTW7 were imaged over a period of $9 \mathrm{~h}$ and 30 min (336 image pairs). A complete stack of $6 z$-section image pairs was acquired every $10 \mathrm{~min} 25 \mathrm{~s}$, resulting in the three-dimensional, time-lapse sequence shown in Figure 3B. The tightly clustered membranes of the Golgi complex labeled by GT-W7 (green) were dynamic over the course of the experiment, frequently extending tubules (Figure 3B inset) and rearranging their architecture. The nuclear envelope underwent partial rotation and indentation in the region of the microtubule organizing center (Figure 3B; time, 5:02: $05)$. There was no indication of damage to the cells by the repetitive laser radiation even after this extended imaging 
experiment. Brightness levels of both markers were virtually identical from the start to the end of the experiment.

In conclusion, the W7/10C GFP variant combination described here allows dual-color, time-lapse imaging in live cells over long periods of time with minimal photobleaching and negligible crossover between the variants. Fusion proteins with $\mathrm{W} 7$ and $10 \mathrm{C}$ as reporter molecules were sufficiently photostable to easily sustain prolonged imaging with high numerical aperture objectives, and sufficiently bright to allow detection of small intracellular structures. They could be detected on a wide-field fluorescence microscope by switching the excitation source alone, making them suitable for double-label imaging on a wide range of microscopes that have computer control of excitation filter wheels, e.g., many CCD cameras, silicon-intensified target (SIT) cameras and regular epifluorescence systems. Using a CLSM with a 413-nm krypton laser source, the brightness of W7 was improved significantly over the wide-field microscope system with crossover reduced below $1 \%$. In addition to allowing extended dual-color, time-lapse imaging, the W7/10C GFP variants and the microscope systems described here have the potential to be used in a wide variety of other applications in living cells. These include ratio imaging experiments (4) to address questions related to protein sorting and degradation, doublelabel fluorescence recovery after photobleaching experiments (FRAP; References 2 and 5) to measure the diffusional mobility of proteins and fluorescence resonance energy transfer (FRET; References 13 and 14) to study protein-protein interactions. We expect such applications are only a few of the numerous ways in which W7/10C GFP variants will be exploited in future work.

\section{ACKNOWLEDGMENTS}

We would like to thank R. Tsien and R. Heim (both at University of California at San Diego, La Jolla, CA, USA) for providing the W7 cDNA, J. Barsony, National Institute of Diabetes and Digestive and Kidney Diseases, National Institutes of Health (NIDDK, $\mathrm{NIH}$ ) for help with the spectral analy- sis, Silke Schumacher (NIDDK, NIH) for help with the DNA sequencing and Paul Millman (Chroma Technologies) for providing the custom-made filter sets free of charge. Brad Bobbitt (Microcosm, Columbia, MD, USA) is gratefully acknowledged for expert installation of the custom additions to our confocal microscope system. J.E. was supported by a predoctoral grant from Boehringer Ingelheim Fonds, Germany.

\section{REFERENCES}

1.Chalfie, M., Y. Tu, G. Euskirchen, W.W. Ward and D.C. Prasher. 1994. Green fluorescent protein as a marker for gene expression. Science 263:802-805

2.Cole, N.B., C.L. Smith, N. Sciaky, M. Terasaki, M. Edidin and J. LippincottSchwartz. 1996. Diffusional mobility of Golgi proteins in membranes of living cells. Science 273:797-801.

3.Dickson, R.M., A.B. Cubitt, R.Y. Tsien and W.E. Moerner. 1997. On/off blinking and switching behaviour of single molecules of green fluorescent protein. Nature 388:355358.

4.Dunn, K.W., S. Mayor, J.N. Myers and F.R. Maxfield. 1994. Applications of ratio fluorescence microscopy in the study of cell physiology. FASEB J. 8:573-582.

5.Edidin, M. 1994. Fluorescence photobleaching and recovery, FPR, in the analysis of membrane structure and dynamics, p. 109-135. In S. Damjanocich, M. Edidin and J. Szollosi (Eds.), Mobility and Proximity in Biological Membranes. CRC Press, Boca Raton, FL.

6.Ellenberg, J., E.D. Siggia, J.E. Moreira, C.L. Smith, J.F. Presley, H.J. Worman and J. Lippincott-Schwartz. 1997. Nuclear membrane dynamics and reassembly in living cells: targeting of an inner nuclear membrane protein in interphase and mitosis. J. Cell Biol. 138:1193-1206.

7.Heim, R., A.B. Cubitt and R.Y. Tsien. 1995. Improved green fluorescence. Nature 373: 663-664.

8.Heim, R., D.C. Prasher and R.Y. Tsien. 1994. Wavelength mutations and posttranslational autoxidation of green fluorescent protein. Proc. Natl. Acad. Sci. USA 91:1250112504.

9.Heim, R. and R.Y. Tsien. 1996. Engineering green fluorescent protein for improved brightness, longer wavelengths and fluorescence resonance energy transfer. Curr. Biol. 6:178-182.

10.Lippincott-Schwartz, J. and C.L. Smith 1997. Insights into secretory and endocytic membrane traffic using green fluorescent protein chimeras. Curr. Opin. Neurobiol. 7:631639.

11.Lippincott-Schwartz, J., L.C. Yuan, J.S. Bonifacino and R.D. Klausner. 1989. Rapid redistribution of Golgi proteins into the ER in cells treated with brefeldin A: evidence for membrane cycling from Golgi to ER. Cell 56:801-813.
12.Misteli, T. and D.L. Spector. 1997. Applications of the green fluorescent protein in cell biology and biotechnology. Nature Biotechnol. 15:961-964.

13.Mitra, R.D., C.M. Silva and D.C. Youvan. 1996. Fluorescence resonance energy transfer between blue-emitting and red-shifted excitation derivatives of the green fluorescent protein. Gene 173:13-17.

14.Miyawaki, A., J. Llopis, R. Heim, J.M. McCaffery, J.A. Adams, M. Ikura and R. Tsien. 1997. Fluorescent indicators for $\mathrm{Ca} 2+$ based on green fluorescent proteins and calmodulin. Nature 388:882-887.

15.Ormo, M., A.B. Cubitt, K. Kallio, L.A. Gross, R.Y. Tsien and S.J. Remington. 1996. Crystal structure of the Aequorea victoria green fluorescent protein. Science 273:1392-1395.

16.Patterson, G.H., S.M. Knobel, W.D. Sharif, S.R. Kain and D.W. Piston. 1997. Use of the green fluorescent protein and its mutants in quantitative fluorescence microscopy. Biophys. J. 73:2782-2790.

17.Prasher, D.C., V.K. Eckenrode, W.W. Ward, F.G. Prendergast and M.J. Cormier. 1992. Primary structure of the Aequorea victoria green-fluorescent protein. Gene 111:229-233.

18.Presley, J.F., N.B. Cole, T.A. Schroer, K. Hirschberg, K.J. Zaal and J. LippincottSchwartz. 1997. ER-to-Golgi transport visualized in living cells. Nature 389:81-85.

19.Rizzuto, R., M. Brini, G.F. De, R. Rossi, R. Heim, R.Y. Tsien and T. Pozzan. 1996. Double labelling of subcellular structures with organelle-targeted GFP mutants in vivo. Curr. Biol. 6:183-188.

20.Rizzuto, R., M. Brini, P. Pizzo, M. Murgia and T. Pozzan. 1995. Chimeric green fluorescent protein as a tool for visualizing subcellular organelles in living cells. Curr. Biol. 5:635642.

21.Sciaky, N., J. Presley, C. Smith, K.J. Zaal, N. Cole, J.E. Moreira, M. Terasaki, E. Siggia and S.J. Lippincott. 1997. Golgi tubule traffic and the effects of brefeldin A visualized in living cells. J. Cell Biol. 139:1137-1155.

22.Wubbolts, R., M. Fernandez-Borja, L. Oomen, D. Verwoerd, H. Janssen, J. Calafat, A. Tulp, S. Dusseljee and J. Neefjes. 1996. Direct vesicular transport of MHC class II molecules from lysosomal structures to the cell surface. J. Cell Biol. 135:611-622.

23.Zimmermann, T. and F. Siegert. 1998. Simultaneous detection of two GFP spectral mutants during in vivo confocal microscopy of migrating Dictyostelium cells. BioTechniques 24:458-461.

Received 2 April 1998; accepted 26 May 1998.

Address correspondence to:

Dr. John Presley

Cell Biology and Metabolism Branch

NICHD, NIH

Bethesda, MD 20892, USA

Internet:jpresley@helix.nih.gov 